\title{
Subsequent Risk of Metabolic Syndrome in Women With a History of Preeclampsia: Data From the Health Examinees Study
}

Jae Jeong Yang ${ }^{1,2}$, Sang-Ah Lee ${ }^{3}$, Ji-Yeob Choi ${ }^{1,4,5}$, Minkyo Song ${ }^{1,2,4}$, Sohee Han ${ }^{1}$, Hyung-Suk Yoon ${ }^{1,2,4}$, Yunhee Lee ${ }^{1,4}$, Juhwan $\mathrm{Oh}^{6}$, Jong-Koo Lee ${ }^{6,7}$, and Daehee Kang ${ }^{1,4,5}$

${ }^{1}$ Department of Preventive Medicine, Seoul National University College of Medicine, Seoul, Korea

${ }^{2}$ Institute of Environmental Medicine, Seoul National University Medical Research Center, Seoul, Korea

${ }^{3}$ Department of Preventive Medicine, Kangwon National University School of Medicine, Kangwon, Korea

${ }^{4}$ Department of Biomedical Sciences, Seoul National University Graduate School, Seoul, Korea

${ }^{5}$ Cancer Research Institute, Seoul National University, Seoul, Korea

${ }^{6} \mathrm{JW}$ LEE Center for Global Medicine, Seoul National University College of Medicine, Seoul, Korea

${ }^{7}$ Department of Family Medicine, Seoul National University College of Medicine, Seoul, Korea

Received July 22, 2014; accepted November 5, 2014; released online March 7, 2015

Copyright (C) 2015 Jae Jeong Yang et al. This is an open access article distributed under the terms of Creative Commons Attribution License, which permits unrestricted use, distribution, and reproduction in any medium, provided the original author and source are credited.

\begin{abstract}
Background: To investigate whether preeclampsia is independently associated with risk of future metabolic syndrome and whether any such primary associations are modified by different ages at first pregnancy.

Methods: Based on the Health Examinees Study, a cross-sectional analysis was conducted. Data of women $(n=49780)$ who had experienced at least 1 pregnancy during their lifetime and had never been diagnosed with any metabolic disorder before their pregnancy were analyzed using multiple logistic regression models. Odds ratios (ORs) and 95\% confidence intervals (CIs) were estimated after adjusting for age, lifestyle characteristics, and reproductive factors. A stratified analysis was also conducted to estimate the extent of the primary association between preeclampsia and future metabolic syndrome by age at first pregnancy.

Results: Women with a history of preeclampsia had significantly increased odds of developing metabolic syndrome (adjusted OR 1.23; 95\% CI, 1.12-1.35), central obesity (adjusted OR 1.36; 95\% CI, 1.25-1.47), elevated blood pressure (adjusted OR 1.53; 95\% CI, 1.41-1.67), or elevated fasting glucose (adjusted OR 1.13; 95\% CI, 1.03-1.25) in later life. In the stratified analysis, women who first became pregnant at ages $>35$ years and had preeclampsia were found to be at significantly increased likelihood of metabolic syndrome later in life (adjusted OR 4.38; 95\% CI, $1.62-11.9)$.
\end{abstract}

Conclusions: Our findings suggest that preeclampsia increases the risk of metabolic syndrome in later life, and late age at first pregnancy can further exacerbate this risk.

Key words: history of preeclampsia; metabolic syndrome; cohort; HEXA; Korea

\section{INTRODUCTION}

Preeclampsia is one of the most common complications of pregnancy. This pregnancy-specific syndrome, characterized by new-onset hypertension along with proteinuria during gestation, occurs in approximately $3 \%-8 \%$ of all pregnancies worldwide. ${ }^{1}$ Preeclampsia is generally regarded as a disease of the first pregnancy, but it frequently recurs in later pregnancies. $^{2-4}$ Preeclampsia increases the risk of maternal mortality, as well as neonatal morbidity and/or mortality. ${ }^{1,5}$ Furthermore, compelling evidence indicates that the long-term effects of preeclampsia are associated with an increased risk of cardiovascular disease in later life. ${ }^{3,6-10}$

The underlying mechanism for the transition from preeclampsia to cardiovascular disease appears to be multifactorial but has not yet been fully elucidated. Previous studies have suggested that preeclampsia is associated with pathophysiological abnormalities, including endothelial dysfunction and systemic hypertension, and metabolic disorders, such as obesity, insulin resistance, hyperglycemia, and diabetes mellitus. ${ }^{3,6,11-14}$ The occurrence of a cluster of these perturbations in multiple metabolic pathways is 
generally known as metabolic syndrome, a condition that is assumed to create a milieu leading to increased risk of cardiovascular disease. ${ }^{15}$ This means that preeclampsia can increase the risk of metabolic syndrome, which may be an ascendant event of future cardiovascular disease.

In the disease trajectory from preeclampsia to cardiovascular disease, metabolic syndrome may play a crucial role as a putative mediator and/or indicator of susceptibility. It is possible that preeclamptic women can easily progress to metabolic syndrome, which is generally defined as a cluster of cardiovascular risk factors characterized by insulin resistance, ${ }^{16,17}$ or that a subclinical susceptibility to future metabolic syndrome may predispose women to preeclampsia. ${ }^{18}$ We therefore hypothesized that preeclampsia may be a risk factor for metabolic alterations that lead to the development of cardiovascular disease later in life. Furthermore, given that female reproductive history is considered a component of the etiology of several chronic diseases, including metabolic syndrome, ${ }^{19}$ the association between preeclampsia and future metabolic syndrome could be mediated by certain pregnancy-related factors, particularly age at first pregnancy. To explore this hypothesis, a cross-sectional study, based on data from a large-scale cohort study, was conducted in a Korean population to investigate: 1) whether preeclampsia is independently associated with increased risk of metabolic syndrome and its components later in life, and 2) whether any such primary associations are modified by age at first pregnancy.

\section{MATERIALS AND METHODS}

\section{Study population}

The present study is based on a large-scale genomic cohort study, the Health Examinees (HEXA) Study, which was conducted in Korea. Middle-aged and elderly people were prospectively recruited for the HEXA study following a standardized study protocol, which was approved by the Ethics Committee of the Korean Health and Genomic Study of the Korean National Institute of Health and the institutional review boards of all participating hospitals. Before entering the study, all participants voluntarily signed an informed consent form. An interview-based questionnaire survey was conducted to collect information on individual characteristics, including socio-demographic factors, disease history, lifestyle, and dietary habits. Biological samples (ie, plasma, serum, buffy coat, blood cells, genomic DNA, and urine) were collected at baseline and stored under stable conditions. Physical examinations and laboratory analyses were performed by trained medical staff.

The study samples were selected as follows: from a total of 85323 subjects aged 40-69 years who participated in the HEXA study between 2004 and 2008, men $(n=28075)$ and nulligravida women $(n=1902)$ were excluded. Eligible women who had experienced at least 1 pregnancy during their lifetime were preliminarily screened $(n=55346)$. Of these, we excluded women for whom the following information was not available: preeclampsia history $(n=1026)$, age at first pregnancy $(n=259)$, past/current history of metabolic disorders (ie, hypertension, hyperlipidemia, or diabetes mellitus; $n=105$ ), and any kind of anthropometry measures (ie, waist circumference, systolic/diastolic blood pressure, and levels of triglyceride, fasting blood glucose, and highdensity lipoprotein [HDL] cholesterol; $n=3307$ ). To avoid the problem of temporal ambiguity, we also excluded women who had been diagnosed with any metabolic disorder (ie, hypertension, hyperlipidemia, or diabetes mellitus) before their first pregnancy $(n=869)$. After exclusions, a total of 49780 women were included in the final analysis.

\section{Definition of history of preeclampsia}

History of preeclampsia was evaluated by posing questions in phases. First, women were classified as either gravida or nulligravida according to their response to the question "Have you ever been pregnant?" Nulligravida women were excluded from the analysis. The gravida women were then asked, "At what age did you first become pregnant?" and "Have you ever been diagnosed with preeclampsia by a doctor in a hospital?" Women who responded "yes" to the last question were assigned to the preeclampsia-positive group; the respondents who answered "no" were assigned to the preeclampsia-negative group. If a woman was unsure of her history of preeclampsia, she was excluded from the analysis.

\section{Definition of metabolic syndrome}

Metabolic syndrome and its individual components were defined according to the National Cholesterol Education Program Adult Treatment Panel III (NCEP-ATP III) criteria. The NCEP-ATP III criteria for metabolic syndrome require the presence of 3 or more of the following ${ }^{20}$ : a waist circumference $\geq 80 \mathrm{~cm}$, systolic blood pressure $\geq 130 \mathrm{~mm} \mathrm{Hg}$ and/or diastolic blood pressure $\geq 85 \mathrm{~mm} \mathrm{Hg}$ or consuming antihypertensive medication, fasting triglyceride levels $\geq 150$ $\mathrm{mg} / \mathrm{dL}$ or consuming antihyperlipidemic medication, fasting plasma glucose $\geq 100 \mathrm{mg} / \mathrm{dL}$ or consuming antidiabetic medication, and fasting HDL cholesterol $<50 \mathrm{mg} / \mathrm{dL}$ or consuming anticholesterol medication.

\section{Statistical analysis}

The chi-squared test and Student's $t$-test were used to compare the basic characteristics of gravida women who had experienced a pregnancy complicated by preeclampsia and those who had not. All results with a $P$-value $<0.05$ were considered statistically significant.

To investigate whether preeclampsia was independently associated with risk of subsequent metabolic syndrome and its individual components, 3 hierarchical multivariate logistic regression models were used to estimate odds ratios (ORs) 
Table 1. Basic characteristics of the study population across metabolic syndrome $(n=49780)$

\begin{tabular}{|c|c|c|c|c|c|}
\hline & $\begin{array}{l}\text { Metabolic syndrome } \\
\qquad(n=12687)\end{array}$ & $\begin{array}{l}\text { Non-metabolic syndrome } \\
\qquad(n=37093)\end{array}$ & $P^{a}$ & $P^{\mathrm{b}}$ & $P^{\mathrm{c}}$ \\
\hline \multicolumn{6}{|l|}{ Life style factors } \\
\hline Age, years & $56.5(7.2)$ & $51.1(7.3)$ & $<0.001$ & $<0.001$ & $<0.001$ \\
\hline$\geq$ High school graduation & $4734(37.3)$ & $22490(60.6)$ & $<0.001$ & $<0.001$ & $<0.001$ \\
\hline Never smokers & $12215(96.3)$ & $35626(96.1)$ & 0.242 & 0.149 & 0.251 \\
\hline Never drinkers & $9399(74.1)$ & $24184(65.2)$ & $<0.001$ & $<0.001$ & $<0.001$ \\
\hline Regular exercisers & $5962(47.0)$ & $19158(51.7)$ & $<0.001$ & $<0.001$ & $<0.001$ \\
\hline \multicolumn{6}{|l|}{ Reproductive factors } \\
\hline History of preeclampsia & $708(5.6)$ & $1882(5.0)$ & $<0.001$ & $<0.001$ & $<0.001$ \\
\hline Age at first pregnancy, years & $24.3(3.2)$ & $25.2(3.2)$ & $<0.001$ & $<0.001$ & $<0.001$ \\
\hline Number of childbirths & $2.8(1.2)$ & $2.3(0.9)$ & 0.853 & 0.320 & 0.439 \\
\hline History of spontaneous abortion & $2963(23.4)$ & $8771(23.7)$ & 0.119 & 0.222 & 0.280 \\
\hline History of artificial abortion & $8269(65.2)$ & $24842(67.0)$ & 0.002 & $<0.001$ & $<0.001$ \\
\hline Breastfeeding experience & $11517(90.8)$ & $31790(85.7)$ & 0.040 & 0.517 & 0.767 \\
\hline Use of oral contraceptives & $3160(24.9)$ & $7213(19.5)$ & 0.006 & $<0.001$ & 0.003 \\
\hline Menopause & $9588(75.6)$ & $18780(50.6)$ & 0.179 & $<0.001$ & $<0.001$ \\
\hline \multicolumn{6}{|l|}{ Anthropometry } \\
\hline Waist circumference, $\mathrm{cm}$ & $85.5(6.9)$ & $77.1(7.3)$ & $<0.001$ & $<0.001$ & $<0.001$ \\
\hline Systolic blood pressure, $\mathrm{mm} \mathrm{Hg}$ & $131.2(15.9)$ & $117.6(14.6)$ & 0.005 & 0.007 & 0.008 \\
\hline Diastolic blood pressure, $\mathrm{mm} \mathrm{Hg}$ & $80.8(9.6)$ & $73.6(9.5)$ & 0.005 & 0.007 & 0.009 \\
\hline Triglycerides, mg/dL & $174.4(97.5)$ & $93.5(49.1)$ & $<0.001$ & $<0.001$ & $<0.001$ \\
\hline Fasting glucose, $\mathrm{mg} / \mathrm{dL}$ & $104.2(29.1)$ & $89.3(15.1)$ & $<0.001$ & $<0.001$ & $<0.001$ \\
\hline HDL cholesterol, $\mathrm{mg} / \mathrm{dL}$ & $47.7(10.2)$ & $58.8(12.1)$ & $<0.001$ & $<0.001$ & $<0.001$ \\
\hline
\end{tabular}

HDL, high-density lipoprotein.

Data are the mean (standard deviation) or $n(\%)$.

aAdjusted $P$ values by age at enrollment.

${ }^{\mathrm{b}}$ Adjusted $P$ values by age at enrollment, age at first pregnancy, number of childbirths, BMI status before pregnancy, history of spontaneous abortion, history of artificial abortion, breastfeeding experience, use of oral contraceptives, menopausal status, and history of preeclampsia.

${ }^{c}$ Adjusted $P$ values by age at enrollment, age at first pregnancy, number of childbirths, BMl status before pregnancy, history of spontaneous abortion, history of artificial abortion, breastfeeding experience, use of oral contraceptives, menopausal status, education level, smoking status, drinking status, physical exercise, and history of preeclampsia.

and $95 \%$ confidence intervals (CIs) accounting for: age at enrollment (Model 1); age at enrollment, age at first pregnancy, number of childbirths, BMI before pregnancy, history of spontaneous abortion, history of artificial abortion, breastfeeding experience, use of oral contraceptives, and menopausal status (Model 2); and age at enrollment, age at first pregnancy, number of childbirths, BMI before pregnancy, history of spontaneous abortion, history of artificial abortion, breastfeeding experience, use of oral contraceptives, menopausal status, education level, smoking status, drinking status, and physical exercise (Model 3). Furthermore, to estimate the primary association between preeclampsia and metabolic syndrome by age at first pregnancy, a stratified analysis (age $>35$ years, age 31-35 years, and age $\leq 30$ years) was conducted in the same manner. In the stratified analysis, to determine whether the subsequent risk of metabolic syndrome increased with age at first pregnancy, trend tests were performed. Interaction effects between preeclampsia and age at first pregnancy on metabolic syndrome and its components were also evaluated using multiplicative interaction terms in the multivariate logistic regression models. All other statistical analyses were performed using SAS software version 9.3 (SAS Institute, Cary, NC, USA).

\section{RESULTS}

Characteristics of study participants regarding lifestyle, reproduction, and anthropometry are summarized in Table 1. When all putative confounders were accounted for, women with metabolic syndrome were more likely to be older, less educated, nondrinkers, and non-regular exercisers, and to have a history of preeclampsia, be younger at their first pregnancy, and have had less experience with artificial abortion $(P<0.001)$ (Table 1). Women diagnosed with preeclampsia tended to be older at first pregnancy (25.4 years vs 24.9 years) and have higher rates of spontaneous $(27.9 \%$ vs $23.3 \%)$ and artificial $(71.2 \%$ vs $66.3 \%)$ abortions; these differences remained statistically significant after adjusting for potential confounders. When all putative confounders were taken into consideration, women diagnosed with preeclampsia were found to have a significantly higher mean waist circumference $(P<0.05)$ (Table 2).

The prevalence of metabolic syndrome and its components among preeclamptic women is shown in Table 3. A total of $27.3 \%$ of preeclamptic women met the criteria for metabolic syndrome. More than one-third of the subjects had central obesity $(51.1 \%)$, elevated blood pressure $(45.1 \%)$, and reduced levels of HDL cholesterol (35.2\%). Accounting for 
Table 2. Comparison of characteristics according to history of preeclampsia

\begin{tabular}{|c|c|c|c|c|c|}
\hline & $\begin{array}{l}\text { History of preeclampsia } \\
\qquad(n=2590)\end{array}$ & $\begin{array}{l}\text { No history of preeclampsia } \\
\qquad(n=47190)\end{array}$ & $P^{a}$ & $P^{b}$ & $P^{c}$ \\
\hline \multicolumn{6}{|l|}{ Life style factors } \\
\hline Age, years & $52.0(7.4)$ & $52.5(7.6)$ & 0.003 & 0.012 & 0.017 \\
\hline$\geq$ High school graduation & $1510(58.3)$ & $25714(54.5)$ & 0.354 & 0.789 & 0.917 \\
\hline Never smokers & 2477 (95.6) & $45364(96.1)$ & 0.041 & 0.275 & 0.811 \\
\hline Never drinkers & $1735(67.0)$ & $31848(67.5)$ & 0.065 & 0.067 & 0.235 \\
\hline Regular exercisers & $1386(53.5)$ & $23734(50.3)$ & 0.011 & 0.022 & 0.024 \\
\hline \multicolumn{6}{|l|}{ Reproductive factors } \\
\hline Age at first pregnancy, years & $25.4(3.2)$ & $24.9(3.3)$ & $<0.001$ & $<0.001$ & $<0.001$ \\
\hline Number of childbirths & $2.4(0.9)$ & $2.4(1.0)$ & 0.654 & 0.614 & 0.653 \\
\hline History of spontaneous abortion & $723(27.9)$ & $11011(23.3)$ & $<0.001$ & $<0.001$ & $<0.001$ \\
\hline History of artificial abortion & $1844(71.2)$ & $31267(66.3)$ & $<0.001$ & $<0.001$ & $<0.001$ \\
\hline Breastfeeding experience & 2207 (85.2) & $41100(87.1)$ & 0.026 & 0.322 & 0.306 \\
\hline Use of oral contraceptives & $589(22.7)$ & $9784(20.7)$ & 0.445 & 0.057 & 0.037 \\
\hline Menopause & $1472(56.8)$ & $26896(57.0)$ & 0.204 & 0.028 & 0.031 \\
\hline \multicolumn{6}{|l|}{ Anthropometry } \\
\hline Waist circumference, $\mathrm{cm}$ & $80.3(8.3)$ & $79.2(8.1)$ & $<0.001$ & $<0.001$ & $<0.001$ \\
\hline Systolic blood pressure, $\mathrm{mm} \mathrm{Hg}$ & $123.3(16.5)$ & $121.0(16.0)$ & 0.119 & 0.159 & 0.162 \\
\hline Diastolic blood pressure, $\mathrm{mm} \mathrm{Hg}$ & $76.8(10.4)$ & $75.4(10.0)$ & 0.211 & 0.263 & 0.268 \\
\hline Triglycerides, $\mathrm{mg} / \mathrm{dL}$ & $114.7(73.8)$ & $114.1(73.9)$ & 0.292 & 0.188 & 0.150 \\
\hline Fasting glucose, $\mathrm{mg} / \mathrm{dL}$ & $93.9(21.4)$ & $93.1(20.6)$ & 0.976 & 0.999 & 0.996 \\
\hline HDL cholesterol, mg/dL & $55.7(12.6)$ & $56.0(12.6)$ & 0.172 & 0.086 & 0.054 \\
\hline
\end{tabular}

HDL, high-density lipoprotein.

Data are the mean (standard deviation) or $n(\%)$.

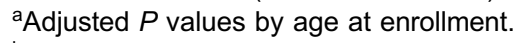

${ }^{b}$ Adjusted $P$ values by age at enrollment, age at first pregnancy, number of childbirths, BMI status before pregnancy, history of spontaneous abortion, history of artificial abortion, breastfeeding experience, use of oral contraceptives, and menopausal status.

${ }^{c}$ Adjusted $P$ values by age at enrollment, age at first pregnancy, number of childbirths, BMl status before pregnancy, history of spontaneous abortion, history of artificial abortion, breastfeeding experience, use of oral contraceptives, menopausal status, education level, smoking status, drinking status, and physical exercise.

Table 3. Risk of the development of metabolic syndrome and its components in women with and without a history of preeclampsia

\begin{tabular}{|c|c|c|c|c|c|}
\hline & $\begin{array}{c}\text { History of } \\
\text { preeclampsia } \\
(n=2590)\end{array}$ & $\begin{array}{c}\text { No history of } \\
\text { preeclampsia } \\
(n=47190)\end{array}$ & $\begin{array}{c}\text { Model 1ª OR } \\
(95 \% \mathrm{Cl})\end{array}$ & $\begin{array}{l}\text { Model } 2^{\mathrm{b}} \text { OR } \\
(95 \% \mathrm{Cl})\end{array}$ & $\begin{array}{c}\text { Model } 3^{\mathrm{c}} \text { OR } \\
(95 \% \mathrm{Cl})\end{array}$ \\
\hline Metabolic syndrome & $708(27.3)$ & $11979(25.4)$ & $1.18(1.07-1.29)$ & $1.22(1.11-1.34)$ & $1.23(1.12-1.35)$ \\
\hline \multicolumn{6}{|l|}{ Individual component of metabolic syndrome } \\
\hline Elevated waist circumference & $1323(51.1)$ & $21609(45.8)$ & $1.30(1.20-1.42)$ & $1.34(1.24-1.46)$ & $1.36(1.25-1.47)$ \\
\hline Elevated blood pressure & $1168(45.1)$ & $17630(37.4)$ & $1.50(1.38-1.63)$ & $1.53(1.40-1.66)$ & $1.53(1.41-1.67)$ \\
\hline Elevated triglyceride & $600(23.2)$ & $10614(22.5)$ & $1.08(0.98-1.18)$ & $1.09(0.99-1.20)$ & $1.09(0.99-1.20)$ \\
\hline Elevated fasting glucose & $583(22.5)$ & $10051(21.3)$ & $1.11(1.01-1.22)$ & $1.13(1.02-1.24)$ & $1.13(1.03-1.25)$ \\
\hline Reduced HDL cholesterol & $911(35.2)$ & $16341(34.6)$ & $1.05(0.96-1.14)$ & $1.05(0.97-1.15)$ & $1.06(0.97-1.15)$ \\
\hline
\end{tabular}

$\mathrm{Cl}$, confidence interval; HDL, high-density lipoprotein; OR, odds ratio.

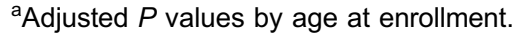

${ }^{\mathrm{b}}$ Adjusted $P$ values by age at enrollment, age at first pregnancy, number of childbirths, BMI status before pregnancy, history of spontaneous abortion, history of artificial abortion, breastfeeding experience, use of oral contraceptives, and menopausal status.

${ }^{c}$ Adjusted $P$ values by age at enrollment, age at first pregnancy, number of childbirths, BMl status before pregnancy, history of spontaneous abortion, history of artificial abortion, breastfeeding experience, use of oral contraceptives, menopausal status, education level, smoking status, drinking status, and physical exercise.

selected covariates, including lifestyle characteristics and reproductive history, women diagnosed with preeclampsia had significantly increased odds of developing metabolic syndrome (OR 1.23; 95\% CI, 1.12-1.35), central obesity (OR 1.36; 95\% CI, 1.25-1.47), elevated blood pressure (OR 1.53; 95\% CI, 1.41-1.67), and elevated fasting glucose levels (OR 1.13 ; 95\% CI, 1.03-1.25) in later life (Table 3).
A stratified analysis was also conducted to account for the potent effect of late age at first pregnancy (Table 4). Almost half of the women with a history of preeclampsia and late age at first pregnancy ( $>35$ years) had metabolic syndrome (43.5\%); these women had a 4.38-times higher likelihood of metabolic syndrome (95\% CI, 1.62-11.9), as evaluated using the fully adjusted models. Furthermore, the respective odds of 
Table 4. Risk estimates for metabolic syndrome and each component of the metabolic syndrome in women with a history of preeclampsia: stratified analysis by age group at first pregnancy

\begin{tabular}{|c|c|c|c|c|c|}
\hline & \multicolumn{3}{|c|}{ Age group at first pregnancy } & \multirow[b]{2}{*}{$P$ for trend } & \multirow[b]{2}{*}{$P$ interaction } \\
\hline & $\begin{array}{l}\leq 30 \text { years old } \\
\text { OR }(95 \% \mathrm{Cl})\end{array}$ & $\begin{array}{c}31-35 \text { years old } \\
\text { OR }(95 \% \mathrm{Cl})\end{array}$ & $\begin{array}{l}>35 \text { years old } \\
\text { OR }(95 \% \mathrm{Cl})\end{array}$ & & \\
\hline \multicolumn{6}{|l|}{ Model $1^{\text {a }}$} \\
\hline Metabolic syndrome & $1.15(1.04-1.26)$ & $1.80(1.12-2.91)$ & $3.76(1.53-9.22)$ & $<0.001$ & 0.007 \\
\hline \multicolumn{6}{|l|}{ Individual component of metabolic syndrome } \\
\hline Elevated waist circumference & $1.27(1.17-1.38)$ & $2.11(1.39-3.19)$ & $3.18(1.31-7.73)$ & $<0.001$ & 0.007 \\
\hline Elevated blood pressure & $1.47(1.35-1.60)$ & $2.31(1.50-3.55)$ & $2.03(0.82-5.05)$ & $<0.001$ & 0.103 \\
\hline Elevated triglyceride & $1.07(0.97-1.18)$ & $1.06(0.63-1.77)$ & $2.64(1.06-6.55)$ & 0.149 & 0.151 \\
\hline Elevated fasting glucose & $1.10(1.00-1.22)$ & $1.38(0.84-2.26)$ & $0.69(0.20-2.41)$ & 0.154 & 0.527 \\
\hline Reduced HDL cholesterol & $1.02(0.94-1.11)$ & $1.34(0.88-2.04)$ & $4.83(2.00-11.7)$ & 0.008 & 0.001 \\
\hline \multicolumn{6}{|l|}{ Model $2^{b}$} \\
\hline Metabolic syndrome & $1.19(1.09-1.32)$ & $1.96(1.20-3.21)$ & $4.26(1.59-11.4)$ & $<0.001$ & 0.008 \\
\hline \multicolumn{6}{|l|}{ Individual component of metabolic syndrome } \\
\hline Elevated waist circumference & $1.32(1.21-1.43)$ & $2.18(1.43-3.35)$ & $3.54(1.36-9.28)$ & $<0.001$ & 0.008 \\
\hline Elevated blood pressure & $1.50(1.38-1.64)$ & $2.40(1.55-3.72)$ & $2.02(0.79-5.18)$ & $<0.001$ & 0.110 \\
\hline Elevated triglyceride & $1.08(0.98-1.20)$ & $1.17(0.69-1.97)$ & $2.50(0.96-6.48)$ & 0.142 & 0.164 \\
\hline Elevated fasting glucose & $1.13(1.02-1.25)$ & $1.40(0.84-2.33)$ & $0.68(0.19-2.52)$ & 0.156 & 0.566 \\
\hline Reduced HDL cholesterol & $1.03(0.94-1.12)$ & $1.46(0.95-2.24)$ & $5.58(2.18-14.3)$ & 0.008 & 0.002 \\
\hline \multicolumn{6}{|l|}{ Model $3^{c}$} \\
\hline Metabolic syndrome & $1.20(1.09-1.32)$ & $2.12(1.29-3.49)$ & $4.38(1.62-11.9)$ & $<0.001$ & 0.007 \\
\hline \multicolumn{6}{|l|}{ Individual component of metabolic syndrome } \\
\hline Elevated waist circumference & $1.32(1.22-1.44)$ & $2.26(1.47-3.46)$ & $3.81(1.44-10.0)$ & $<0.001$ & 0.006 \\
\hline Elevated blood pressure & $1.51(1.38-1.64)$ & $2.41(1.55-3.74)$ & $1.97(0.77-5.09)$ & $<0.001$ & 0.116 \\
\hline Elevated triglyceride & $1.08(0.98-1.20)$ & $1.25(0.74-2.11)$ & $2.52(0.96-6.61)$ & 0.104 & 0.153 \\
\hline Elevated fasting glucose & $1.13(1.03-1.25)$ & $1.46(0.87-2.44)$ & $0.64(0.17-2.39)$ & 0.098 & 0.554 \\
\hline Reduced HDL cholesterol & $1.03(0.95-1.13)$ & $1.47(0.95-2.27)$ & $5.50(2.12-14.3)$ & 0.006 & 0.002 \\
\hline
\end{tabular}

$\mathrm{Cl}$, confidence interval; $\mathrm{HDL}$, high-density lipoprotein; OR, odds ratio.

${ }^{a}$ Adjusted $P$ values by age at enrollment.

${ }^{\text {b} A d j u s t e d ~} P$ values by age at enrollment, age at first pregnancy, number of childbirths, BMI status before pregnancy, history of spontaneous abortion, history of artificial abortion, breastfeeding experience, use of oral contraceptives, and menopausal status.

${ }^{\mathrm{c} A d j u s t e d} P$ values by age at enrollment, age at first pregnancy, number of childbirths, BMI status before pregnancy, history of spontaneous abortion, history of artificial abortion, breastfeeding experience, use of oral contraceptives, menopausal status, education level, smoking status, drinking status, and physical exercise.

central obesity and reduced HDL cholesterol level later in life were 3.81 times $(95 \% \mathrm{CI}, 1.44-10.0)$ and 5.50 times $(95 \% \mathrm{CI}$, 2.12-14.3) higher among these women than in those with preeclampsia who became pregnant at younger ages. Even when all putative covariates were accounted for, preeclamptic women who first became pregnant between the age of 31 and 35 years were twice as likely to develop metabolic syndrome (OR 2.12; 95\% CI, 1.29-3.49), central obesity (OR 2.26; 95\% CI, 1.47-3.46), and elevated blood pressure (OR 2.41; 95\% CI, 1.55-3.74). Compared to women who first became pregnant at younger ages, preeclamptic women who first became pregnant at age $>35$ years tended to have higher odds of developing metabolic syndrome in later life. The subsequent risk of metabolic syndrome, central obesity, and elevated blood pressure increased with age at first pregnancy $(P$ for trend $<0.001)$. Significant interaction effects were observed between preeclampsia and age at first pregnancy in terms of the development of metabolic syndrome, central obesity, and reduced HDL cholesterol $(P=0.007, P=0.006$, and $P=0.002$, respectively) (Table 4 ).

\section{DISCUSSION}

A history of preeclampsia appears to be independently associated with increased odds of metabolic syndrome and its individual components, including central obesity, elevated blood pressure, and elevated fasting glucose level, in women after their first pregnancy. Additionally, women who first became pregnant at older ages and had preeclampsia were found to be at significantly increased likelihood of developing metabolic syndrome later in life.

Preeclampsia itself may contribute to subsequent risk of pathophysiological abnormalities related to cardiovascular diseases, which occur due to perturbations in multiple metabolic pathways. Although the sample in the present study was restricted to healthy gravida women who were free of any pre-existing metabolic disorders, they were found to be significantly more prone to developing metabolic disorders, such as central obesity, elevated blood pressure, elevated fasting glucose level, and other such conditions in later life, simply by having a pregnancy complicated by preeclampsia. 
Preeclamptic women are assumed to be at greater risk of poor health as they age, since metabolic syndrome is a crucial risk factor for cardiovascular disease incidence and mortality ${ }^{21}$ and the prevalence of metabolic syndrome tends to increase linearly with age, due to multiple age-related physiologic mechanisms. ${ }^{22}$ Further longitudinal follow-up studies will help to determine the long-term effect of preeclampsia and elucidate hidden associations between preeclampsia, cardiovascular disease, and metabolic syndrome.

The present results are consistent with previous studies reporting that preeclamptic women tended to have higher BMI and blood pressure and unfavorable lipid profiles, which are known to be risk factors for cardiovascular disease in later life, ${ }^{23,24}$ and that the prevalence of metabolic syndrome was significantly higher in women who had experienced a pregnancy complicated by preeclampsia. ${ }^{18,25}$ Additionally, a previous meta-analysis reported that women diagnosed with hypertensive pregnancy disorders, such as preeclampsia, eclampsia, and gestational hypertension, were at a higher risk of having subsequent poor biochemical cardiovascular indicators (ie, levels of triglycerides, HDL cholesterol, glucose, insulin, and microalbumin) than women with normotensive pregnancies. ${ }^{26}$ This indicates that abnormal metabolic alterations activated and/or induced by a preeclamptic pregnancy may affect the development of cardiovascular diseases in the future. Further, given that pregnancy is a metabolic and vascular 'stress test' for women, ${ }^{14}$ a woman diagnosed with preeclampsia (ie, a woman who fails the stress test) may be assumed to be more prone to cardiovascular-related complications caused by metabolic disorders over her lifetime than women with normal pregnancies. ${ }^{14,16}$

Advanced maternal age appears to provoke a negative effect of preeclampsia on risk of subsequent development of metabolic syndrome. In the present study, we attempted to evaluate both the independent and synergistic effects of preeclampsia and late age at first pregnancy on subsequent metabolic disease risks over the lifetime of gravida women. In contrast with preeclampsia, maternal age itself was found to be inversely associated with risk of metabolic syndrome later in life (data not shown). This is consistent with previous findings from Korea that postmenopausal women who had their first child at an older age were at lower risk of developing metabolic syndrome. ${ }^{19}$ This finding is probably best explained by the fact that advanced maternal age is associated with decreased parity and/or number of pregnancies; therefore, women who first become pregnant at older ages are less likely to be exposed to the detrimental effects (ie, insulin resistance, weight gain, and dyslipidemia) that can be induced by accumulated pregnancies, thereby reducing the risk of metabolic syndrome in later life. ${ }^{19,27,28}$

Interestingly, our results show that the protective effects of late age at first pregnancy on metabolic health in middle-aged women dissipate if the women become pregnant at an older age and experience a pregnancy complicated by preeclampsia. Preeclamptic women who first became pregnant at age $>35$ years were found to have a significant increase in their adjusted risk of metabolic syndrome compared to women younger than 35 at first pregnancy without preeclampsia. Although the results were somewhat hindered by the small number of women exposed to both conditions (ie, preeclampsia and late age at first pregnancy), our findings indicate that preeclampsia and late age at first pregnancy could be linked and act as crucial risk factors in both a dependent and/or independent manner in the etiology of future metabolic syndrome. Experimental and longitudinal studies focused on biological changes and interactions in the female reproductive system during pregnancy will further clarify the causal mechanisms underlying the long-term effects of maternal age, preeclampsia, and future metabolic health in women.

A certain number of metabolic and vascular alterations generally occur due to the developing placenta and fetus; however, preeclamptic women who also have endothelial damage and dysfunction tend to be under excessive metabolic stress. ${ }^{16}$ Moreover, the features of preeclampsia, including insulin resistance, hypertension, and unfavorable lipid profiles, overlap with those of metabolic syndrome. This implies that the basis of preeclampsia depends on metabolic disturbances and that the excessive metabolic stress that occurs during gestation could be retained and/or turn into metabolic syndrome after pregnancy. Based on a literature review $^{11-14,16,21,24}$ and the present results, it is possible to infer that: 1) metabolic stress, whether it is a new-onset condition induced by pregnancy or an intensified predisposition throughout pregnancy, appears to be an overt antecedent of metabolic syndrome in later life; 2) metabolic stress during pregnancy in women who first become pregnant at an age over 35 years is more likely to have a severe impact on future metabolic health; and 3) women who have both a history of preeclampsia and first pregnancy at an older age may be vulnerable and more likely to experience metabolic syndrome and cardiovascular disease in the future.

Several limitations to this study should be noted. First, preeclampsia ascertainment was based on self-reported information; therefore, this study may not be completely free of misclassification and/or recall bias. If a large proportion of the preeclampsia-positive women were missed, or if silent cases were misclassified into the preeclampsianegative group, the results could be biased. However, given that these effects would tend toward the null hypothesis and attenuate the magnitude of risk estimates in the observed associations, the findings of the present study should be relatively conservative estimates of risks. Furthermore, to ensure the reliability of the self-reported information, percent agreement of 570 subjects who re-visited within 4 years after the first enrollment was analyzed. The percent agreements of preeclampsia history and parity were relatively high $(94 \%$ and $96 \%$, respectively), and those of other reproductive factors 
reached around $80 \%$ or more, signifying a fairly high level of reliability. Second, we could not explore the pathophysiology of preeclampsia due to a lack of detailed information on preeclampsia, including severity, time of onset, and other comorbid symptoms. Moreover, though the subsequent risk of metabolic syndrome might be affected by various pregnancyrelated disorders as well as preeclampsia itself, other pregnancy complications (ie, eclampsia, gestational diabetes mellitus, and hypertension) could not be assessed in the present study. Therefore, our findings should be interpreted with caution. Finally, the cross-sectional design did not allow us to firmly establish a causal relationship. However, we followed the exposed (ie, preeclampsia-positive) and nonexposed (ie, preeclampsia-negative) subjects over time, and after confirming the development of new-onset metabolic diseases, we selected women who did not have pre-existing metabolic disorders before their first pregnancy and estimated all associations as multivariable odds ratios. Therefore, temporal ambiguity - the major flaw of cross-sectional study designs - is diminished and our results have logical plausibility.

In the present study, we demonstrated that preeclampsia in women who first become pregnant at an older age could increase the risk of developing metabolic syndrome later in life. To screen women who are at high risk of metabolic disorders and cardiovascular diseases later in life, clinicians should identify women who have experienced preeclamptic pregnancy or who first become pregnant at older ages. Women who fit both of these criteria should be treated as having an increased risk of future cardiovascular disease. Our findings suggest that, to promote the cardiovascular and metabolic health of women, these women at increased risk should be targeted for both tailor-made interventions for risk modification and closer surveillance.

\section{ACKNOWLEDGMENTS}

This study was supported by the National Genome Research Institute, Korea Center for Disease Control and Prevention. We would like to thank the participants and all members of the HEXA Study Group.

Conflicts of interest: None declared.

\section{REFERENCES}

1. Hutcheon JA, Lisonkova S, Joseph KS. Epidemiology of preeclampsia and the other hypertensive disorders of pregnancy. Best Pract Res Clin Obstet Gynaecol. 2011;25:391-403.

2. Hernández-Díaz S, Toh S, Cnattingius S. Risk of pre-eclampsia in first and subsequent pregnancies: prospective cohort study. BMJ. 2009;338:b2255.

3. Wilson BJ, Watson MS, Prescott GJ, Sunderland S, Campbell DM, Hannaford P, et al. Hypertensive diseases of pregnancy and risk of hypertension and stroke in later life: results from cohort study. BMJ. 2003;326:845.

4. Sibai B, Dekker G, Kupferminc M. Pre-eclampsia. Lancet. 2005;365:785-99.

5. Report of the National High Blood Pressure Education Program Working Group on High Blood Pressure in Pregnancy. Am J Obstet Gynecol. 2000;183:S1-22.

6. Harskamp RE, Zeeman GG. Preeclampsia: at risk for remote cardiovascular disease. Am J Med Sci. 2007;334:291-5.

7. Bellamy L, Casas JP, Hingorani AD, Williams DJ. Pre-eclampsia and risk of cardiovascular disease and cancer in later life: systematic review and meta-analysis. BMJ. 2007;335:974.

8. Craici IM, Wagner SJ, Hayman SR, Garovic VD. Pre-eclamptic pregnancies: an opportunity to identify women at risk for future cardiovascular disease. Womens Health (Lond Engl). 2008;4: 133-5.

9. McDonald SD, Malinowski A, Zhou Q, Yusuf S, Devereaux PJ. Cardiovascular sequelae of preeclampsia/eclampsia: a systematic review and meta-analyses. Am Heart J. 2008;156:918-30.

10. Lykke JA, Langhoff-Roos J, Sibai BM, Funai EF, Triche EW, Paidas MJ. Hypertensive pregnancy disorders and subsequent cardiovascular morbidity and type 2 diabetes mellitus in the mother. Hypertension. 2009;53:944-51.

11. Berends AL, de Groot CJ, Sijbrands EJ, Sie MP, Benneheij SH, Pal R, et al. Shared constitutional risks for maternal vascularrelated pregnancy complications and future cardiovascular disease. Hypertension. 2008;51:1034-41.

12. Valdiviezo C, Garovic VD, Ouyang P. Preeclampsia and hypertensive disease in pregnancy: their contributions to cardiovascular risk. Clin Cardiol. 2012;35:160-5.

13. Ramsay JE, Stewart F, Greer IA, Sattar N. Microvascular dysfunction: a link between pre-eclampsia and maternal coronary heart disease. BJOG. 2003;110:1029-31.

14. Newstead J, von Dadelszen P, Magee LA. Preeclampsia and future cardiovascular risk. Expert Rev Cardiovasc Ther. 2007;5: 283-94.

15. Deedwania PC. Metabolic syndrome and vascular disease: is nature or nurture leading the new epidemic of cardiovascular disease? Circulation. 2004;109:2-4.

16. Rodie VA, Freeman DJ, Sattar N, Greer IA. Pre-eclampsia and cardiovascular disease: metabolic syndrome of pregnancy? Atherosclerosis. 2004;175:189-202.

17. Sattar N, Greer IA. Pregnancy complications and maternal cardiovascular risk: opportunities for intervention and screening? BMJ. 2002;325:157-60.

18. Lu J, Zhao YY, Qiao J, Zhang HJ, Ge L, Wei Y. A follow-up study of women with a history of severe preeclampsia: relationship between metabolic syndrome and preeclampsia. Chin Med J (Engl). 2011;124:775-9.

19. Cho GJ, Park HT, Shin JH, Kim T, Hur JY, Kim YT, et al. The relationship between reproductive factors and metabolic syndrome in Korean postmenopausal women: Korea National Health and Nutrition Survey 2005. Menopause. 2009;16: 998-1003.

20. Grundy SM, Cleeman JI, Daniels SR, Donato KA, Eckel RH, Franklin BA, et al. Diagnosis and management of the metabolic syndrome: an American Heart Association/National Heart, Lung, and Blood Institute Scientific Statement. Circulation. 2005;112: 2735-52. 
21. Galassi A, Reynolds K, He J. Metabolic syndrome and risk of cardiovascular disease: a meta-analysis. Am J Med. 2006;119: $812-9$.

22. Bechtold M, Palmer J, Valtos J, Iasiello C, Sowers J. Metabolic syndrome in the elderly. Curr Diab Rep. 2006;6:64-71.

23. Magnussen EB, Vatten LJ, Smith GD, Romundstad PR. Hypertensive disorders in pregnancy and subsequently measured cardiovascular risk factors. Obstet Gynecol. 2009;114: 961-70.

24. Romundstad PR, Magnussen EB, Smith GD, Vatten LJ. Hypertension in pregnancy and later cardiovascular risk: common antecedents? Circulation. 2010;122:579-84.

25. Smith GN, Pudwell J, Walker M, Wen SW. Risk estimation of metabolic syndrome at one and three years after a pregnancy complicated by preeclampsia. J Obstet Gynaecol Can. 2012;34: 836-41.

26. Hermes W, Ket JC, van Pampus MG, Franx A, Veenendaal MV, Kolster C, et al. Biochemical cardiovascular risk factors after hypertensive pregnancy disorders: a systematic review and metaanalysis. Obstet Gynecol Surv. 2012;67:793-809.

27. Cohen A, Pieper CF, Brown AJ, Bastian LA. Number of children and risk of metabolic syndrome in women. J Womens Health (Larchmt). 2006;15:763-73.

28. Wen W, Gao YT, Shu XO, Yang G, Li HL, Jin F, et al. Sociodemographic, behavioral, and reproductive factors associated with weight gain in Chinese women. Int J Obes Relat Metab Disord. 2003;27:933-40. 